Retinal noise, the performance of retinal ganglion cells, and visual sensitivity in the dark-adapted frog

\author{
Aho, A.-C. \\ Optical Society of America \\ 1987
}

Journal of the Optical Society of America. 1987. 4: 2321-2329

http://hdl.handle.net/1975/958

Downloaded from Helda, University of Helsinki institutional repository.

This is an electronic reprint of the original article.

This reprint may differ from the original in pagination and typographic detail.

Please cite the original version. 


\title{
Retinal noise, the performance of retinal ganglion cells, and visual sensitivity in the dark-adapted frog
}

\author{
A.-C. Aho, K. Donner, C. Hydén and T. Reuter \\ Department of Zoology, University of Helsinki, Helsinki SF-00100, Finland \\ O. Yu. Orlov \\ Institute of Problems of Information Transmission, Academy of Sciences of the USSR, 103051 Moscow K-51, \\ USSR
}

Received August 3, 1987; accepted August 20, 1987

\begin{abstract}
By extracellular recording of action potentials from single ganglion cells in the dark-adapted eyecup of the frog, we show that four different characteristics of a cell, the frequency-of-response function, the variability in responses to low suprathreshold stimulation, the maintained discharge, and the susceptibility to desensitization by weak background lights, all seem to reflect the same intrinsic noise. In a small fraction of reliable cells this noise is close to that expected from dark isomerizationlike events in rods, but it differs by orders of magnitude between cells. We have also determined a threshold intensity for the phototactic jumping behavior of frogs in darkness. This intensity, while below the human threshold, is still above the threshold for an appreciable fraction of frog retinal ganglion cells, and the sensitivity of this visually guided behavior at $16^{\circ} \mathrm{C}$ is not strictly limited by dark events in rods.
\end{abstract}

\section{INTRODUCTION}

The idea that the absolute sensitivity of vision is limited by an intrinsic dark light was originated by Fechner in the middle of the nineteenth century. ${ }^{1}$ After the realization that the absolute sensitivity of humans is so low that the threshold performance must necessarily be influenced by quantal fluctuations ${ }^{2}$ and after the experimental demonstration of this by Hecht et al., ${ }^{3}$ the dark light could be given a more specific formulation by Barlow ${ }^{4}$ as a photonlike noise interfering with the detection of a light signal. This idea gained overwhelming credibility when Ashmore and Falk ${ }^{5}$ found direct evidence for this type of rod noise in intracellular recordings from dark-adapted dogfish bipolar cells, and Baylor and his colleagues ${ }^{6,7}$ found spontaneous electric (dark) events that were indistinguishable from the events generated by real photoisomerizations in the rods of the toad and the monkey. The rate of such events in the monkey was found to be consistent with many psychophysical estimates of human dark light.

The evidence for this agreement between noise at the input and noise at the output of the visual system is not strong, however, since psychophysical estimates vary by two orders of magnitude. ${ }^{8}$ We therefore set out to test the hypothesis that performance at the absolute threshold is limited by the dark rod events step by step through the retina up to behavior. This is not possible with humans, but it can be done with anuran amphibians (frogs and toads), from which there is also direct information on the rate of such events. ${ }^{6}$

In previous studies we concluded that membrane noise in the distal retina (in the horizontal cells of the intact, darkadapted toad eyecup) is indeed consistent with the measured rate of dark events in isolated rods and that the threshold response reliability of the most sensitive retinal ganglion cells is limited by a noise that is likewise consistent with that rate in both the toad (at $20^{\circ} \mathrm{C}$ ) and in the frog (at $\left.11.5^{\circ} \mathrm{C}\right) .{ }^{9-12}$

In the present paper we relate the extracellularly studied performance of single ganglion cells in the frog retina to the absolute sensitivity of the phototactic behavior of the freely moving frog. We first show that frequency-of-response (FOR) experiments, which have been our main tool for assessing ganglion-cell performance, really measure biologically relevant noise, reflected in several important aspects of the cell's performance: the variability of suprathreshold responses, the maintained discharge, and the susceptibility to desensitization by backgrounds. We then consider behavioral sensitivity in the light of the known properties of the ganglion-cell population. We find an approximate agreement between the performance of sensitive single cells and that of the whole animal.

\section{MATERIALS AND METHODS}

All the experiments were done on the common European frog Rana temporaria L. The animals were caught in the autumn in southern Finland or near Moscow (for the behavioral studies there) and were kept in hibernating conditions (basins at $4^{\circ} \mathrm{C}$ in near-darkness and without feeding) until the evening before an electrophysiological experiment or the morning of a behavioral experiment.

\section{Extracellular Ganglion-Cell Recording}

Dissection, recording, and stimulation techniques have been described elsewhere, as has the calculation of light absorbed in red rods and the determination of response thresholds. $9,10,13-15$ The only new feature here is that the experiments were recorded on tape in their entirety so that the maintained discharge could be assessed accurately after- 
ward. The response patterns were transferred as trains of dots (see Fig. 2 below) from the tape to the screen of a storage oscilloscope after each experiment. Before that the shape of each spike potential was visually checked on the screen of another storage oscilloscope to ascertain that only real spikes from one and the same cell were accepted into the dot patterns or into the count of maintained activity. Usually, this posed no problem, as the isolation of units was good. All results refer to flash responses or on-responses of cells representing classes 1 and 2 (not distinguished) or class $3 .{ }^{16-18}$

\section{Summation Parameters}

Background lights, whether real or dark, represent rates of events; in order to transform these into numbers of events determining the Poisson statistics at the ganglion cell, we must know the summation characteristics of that cell. The summation area $A_{s}$ (the size of the receptive field) is here given in the top-hat approximation; it is determined as the ratio of the threshold quantity of light delivered with a small spot of area $A_{0} \ll A_{s}$ to the threshold density of light (quanta absorbed per square millimeter) delivered with a spot larger than the receptive field. If $I_{0}$ (small) and $I_{L}$ (large) are the threshold intensities of equal-duration stimuli, then $A_{s}=$ $A_{0} I_{0} / I_{L} \cdot{ }^{15,17-19}$ (A prerequisite is that the large spot does not activate the inhibitory surround so much that $I_{L}$ is raised by inhibition. This source of error can be avoided mainly by the use of only moderately large spots; even in a strongly light-adapted eye, the integrated spatial sensitivity distribution of a cell may then be underestimated by, at the most, some $20 \% .{ }^{18}$ In fully dark-adapted frog ganglion cells, as studied here, the threshold-raising capacity of lateral inhibition is much weaker. ${ }^{20}$ )

Often it is advantageous to express the degree of spatial summation in terms of the number of rods contained within $A_{s}$; this is achieved by multiplying $A_{s}$ (in square millimeters) by the rod density in the retina of the common frog, 15,700 $\mathrm{mm}^{-2} \cdot$. $^{21}$

Similarly, the summation or integration time $t_{i}$ is here given as a sharply delimited time window, whereby it is determined as the ratio of the threshold quantity of light delivered with a brief flash of duration $t_{f} \ll t_{i}$ to the threshold flux of light $q$ (in reciprocal seconds) delivered with a step stimulus. If $I_{f}$ (flash) and $I_{1}$ (step) are the threshold intensities of spatially coextensive stimuli, then $t_{i}=t_{f} I_{f} /$ $I_{1} \cdot 9,15,22$

The accuracy of these estimates of $A_{s}$ and $t_{i}$ depends entirely on the accuracy of the threshold determinations. A rough error estimate can be obtained as follows. Each determination of the (log) threshold was based on at least 10 20 stimulus presentations (some, in fact, were based on complete FOR experiments; see below); experience shows that, in the great majority of cells, this fixes the threshold within $0.1 \log$ unit. The sampling distributions of $\log A_{s}$ and $\log t_{i}$ (both depending on a difference between log thresholds) are broader by a factor of $2^{1 / 2} \approx 1.4$ and that of $\log A_{s} t_{i}$ (total summation) is broader by a factor of 2 . Accordingly, $\log A_{s} t_{i}$ has a precision of roughly $0.2 \mathrm{log}$ unit, and so the estimates of $A_{s} t_{i}$ would lie between 0.6 and 1.6 times the real value. Note that this uncertainty does not pertain to the numbers of stimulus-induced (flash-induced) isomerizations collected by the ganglion cell (see below), because our stimulus flashes were always much smaller than $A_{s}$ and much shorter than $t_{i}$.
Rates and Numbers of Light-Induced, Dark, and

Noise-Equivalent Dark Events

Only isomerizations falling within the summation area and within one summation time of the cell can be confused with a brief flash stimulus. Hence, from the viewpoint of flash detection, a background rate of isomerization events can be translated into a number of events by multiplication with $A_{s} t_{i}$. Conceptually, we distinguish between three types of such events: (1) photoisomerizations, for which we denote by $N$ the mean number of photoisomerizations delivered by a stimulus flash $\left(N_{t}\right.$ is then the threshold number eliciting responses on half the trials) and by $B$ the mean number summed from a background light by the ganglion cell within $A_{s}$ and $t_{i} ;(2)$ dark isomerizationlike events in rods, for which the expected rate as calculated from the results of Baylor et al. ${ }^{6}$ corrected for the size of frog rods, is 0.006 per rod per sec at $11.5^{\circ} \mathrm{C}$ (the temperature of the ganglion-cell recordings) and 0.010 per rod per sec at $16^{\circ} \mathrm{C}$ (the temperature of the behavioral experiments), and we denote by $D$ the number of such events summed by the ganglion cell within $A_{s}$ and $t_{i} ;$ (3) noise-equivalent dark events in rods. Following Barlow, ${ }^{4}$ we choose to express all intrinsic retinal noise affecting a ganglion cell in terms of the rate of isomerization events in rods that would be equally detrimental to flash detection (degrade the signal/noise ratio by the same factor, see below), and we denote by $X$ the number of such events summed by the ganglion cell within $A_{s}$ and $t_{i}$. Note that $X$ thus defined denotes a total number of dark events in rods, including $D$.

$N, B, D$, and $X$ are all mean numbers of isomerizations (or isomerizationlike) events within $A_{s}$ and $t_{i}$. The rate of such events is usually expressed in units of $\mathrm{Rh}^{*} \sec ^{-1}$, where $\mathrm{Rh}^{*}$ denotes 1 isomerization/rod.

\section{Frequency-of-Response Experiments}

Our basic method for estimating the noise affecting a cell is the FOR experiment. This is an application to ganglion cells of the classical psychophysical frequency-of-seeing experiment. ${ }^{3,4}$ The subject (the cell) is presented with stimulus flashes at a few mixed mean intensities just around the threshold a great number of times in random order, and the proportion of the trials reported as seen is recorded. A frog ganglion cell, which has a low rate of maintained activity (see below), reports seeing by generating one or several spikes; we regarded as a response the occurrence of at least one spike within a time window $0.5-3$ sec after the flash. A plot of these relative frequencies against the log mean number of flash-induced isomerizations is a frequency of response function. All our FOR functions are based on at least 12 presentations of the stimulus at each mean intensity. (An illustration of how original data are transformed into FOR functions can be seen in Fig. 2 below.)

These functions are analyzed on the assumption that the cell gives off one or more spikes precisely when the total number of isomerization (or isomerizationlike) events summed by the cell exceeds a criterion level $c$. If those events are either flash-induced isomerizations (with a known mean $N$ ) or noise-equivalent events (with an unknown mean $X$ ), the FOR function is expected to follow the cumulative Poisson probabilities that the actual event number $n+x$ with a mean $N+X$ will exceed $c$; i.e., $f(N)=P(n+$ $x \geq c)$. The shape of this function reflects the two parameters $c$ and $X$ : the smaller $c$ is and/or the larger $X$ is, the 
shallower the curve will be. ${ }^{23}$ For us, however, there is essentially only one unknown parameter, $X$, because $c, X$, and the recorded $50 \%$ threshold number of flash-induced isomerizations $N_{t}$ are related: $N_{t}=c-X$. Therefore we can estimate the noise-equivalent event number by finding (on a computer) the $X$ value that gives a maximum-likelihood fit of the cumulative Poisson curve to the data points. ${ }^{9,10,12}$

\section{Signal/Noise Ratio and Quantum Efficiency}

The signal/noise ratio (SNR) in general is defined as the signal mean divided by the standard deviation. Upper limits to the reliability of flash responses can then be calculated as the input SNR $\left(\mathrm{SNR}_{\text {in }}\right)$ from the physical stimulus..$^{9,10,12}$ In our case, the signal is always $N$ flash-induced isomerizations, whereas the (Poisson) standard deviations are square roots of the mean total number of events summed with the flash in the various situations: $N$ (the physical stimulus in darkness), $N+D$ (if dark rod events are treated as a real light, part of the physical stimulus), $N+B$ (the physical stimulus when a background light is shining), or $N+B+D$ (if the dark rod events are included in the background situation). In full darkness we thus obtain

$$
\mathrm{SNR}_{\text {in }}=N / N^{1 / 2}=N^{1 / 2}
$$

if only the actual physical stimulus is considered, or, if the dark rod events are treated as part of the physical stimulus situation,

$$
\mathrm{SNR}_{\text {in }}=N /(N+D)^{1 / 2} .
$$

The physiologically realized output $\mathrm{SNR}, \mathrm{SNR}_{\text {out }}$, can be written by analogy, utilizing the noise-equivalent event number $X$ determined, e.g., by FOR experiments. In darkness,

$$
\mathrm{SNR}_{\text {out }}=N /(N+X)^{1 / 2} .
$$

The degree to which a cell approaches the performance of a perfect detector can be expressed as the quantum efficiency $\mathrm{QE},{ }^{24}$ where

$$
\mathrm{QE}=100 \times\left(\mathrm{SNR}_{\text {out }}\right)^{2} /\left(\mathrm{SNR}_{\text {in }}\right)^{2} .
$$

In darkness this becomes [from Eqs. (1) and (3)]

$$
\mathrm{QE}=100 \times N /(N+X) .
$$

We also define a relative quantum efficiency $\mathrm{QE}_{\mathrm{rel}}$, relating the cell's performance to that of a perfect detector with the same summation parameters, detecting a flash against a real background producing an isomerization rate equal to the dark rate reported from single rods [from Eqs. (2)-(4)]:

$$
\mathrm{QE}_{\mathrm{rel}}=100 \times(N+D) /(N+X) .
$$

\section{Measuring Behavioral Sensitivity: Phototaxis}

The frog was placed in a rectangular $16 \mathrm{~cm} \times 28 \mathrm{~cm}$ black box $22 \mathrm{~cm}$ deep with four symmetrically placed circular windows $(4.4 \mathrm{~cm}$ in diameter) in the roof. One of the windows was covered by a mat glass and could be lit from above with different intensities of diffuse 525-nm light; the others were blinds. A jump against a window produced an electric pulse to a counter by closing a mechanical switch. Three hours before the experiment started, the frog was placed (to adapt) in full darkness; the temperature then was the same as in the actual experiment, $16^{\circ} \mathrm{C}$. The experiment for one frog at one intensity lasted $3 \mathrm{~h}$; if the frog did not jump against the lit window within this time, the intensity was considered to be subthreshold. The threshold determination is based on a total of 75 sessions with four frogs each. The threshold values that we report lie within $1 \log$ unit from the intensity at which jumping ceased almost completely. These values still correspond to significant oriented jumping toward the lit window with only an occasional jump recorded against the dark windows. (In fact, unoriented jumping became more frequent only at high intensities of the lit window.)

Estimation of retinal illumination was performed as follows. The eyes of a sitting frog were $4 \mathrm{~cm}$ above the floor of the box, and so the light incident from the window upon a surface $18 \mathrm{~cm}$ below the roof was measured (Airam UVM- 8 calibrated radiometer). Pupil size was determined by photographing several thoroughly dark-adapted frogs face-on and measuring areas from the photographs; the mean area of one pupil in this frontal projection was $10.0 \pm 0.7 \mathrm{~mm}^{2}$. The total corneal reflection of light falling on this whole area was estimated at $9 \%$, by taking the reflection at perpendicular incidence to be $3.5 \%$ and assuming that reflection depends on the angle of incidence in the same way that it would at a water surface. The mean eye diameter of our frog population was $6.57 \pm 0.08 \mathrm{~mm}$. Scaling down the model eye of du Pont and de Groot ${ }^{25}$ (Rana esculenta; eye diameter, 8 $\mathrm{mm}$ ), we arrive at a posterior focal length of $4.48 \mathrm{~mm}$ and a numerical aperture of 0.53 . The image of the window on the retina would thus be $0.92 \mathrm{~mm}^{2}$.

\section{RESULTS}

\section{Performance of Ganglion Cells in Frequency-of-Response Experiments}

\section{Distribution of Quantum Efficiencies in the Population of Cells}

Figure 1 summarizes, as distributions of QE's, ganglion-cell performance at the $50 \%$ response threshold in a simple flashdetection task for a subpopulation of 19 cells sampled by our microelectrodes. The underlying estimates of intrinsic noise as equivalent numbers of photoisomerizations $X$ and response thresholds $N_{t}$ were obtained from FOR recordings (see the Methods section). The performance of the cells is characterized in terms of absolute [Fig. 1(a), QE from Eq. (5)] and relative [Fig. 1(b), $\mathrm{QE}_{\text {rel }}$ from Eq. (6)] quantum efficiencies at the threshold. The experiments and the actual estimates of equivalent noise for the same sample of cells are considered elsewhere. ${ }^{12}$ The main advantage of this alternative presentation is that the measure of quantum efficiency puts noise power in relation to the cell's threshold; the functional significance of a certain noise level, of course, largely depends on the sensitivity of the cell.

Figure 1(a) thus shows how close the cells get to the performance of a perfect physical quantum detector, displayed as a histogram of cells on $10 \%$ intervals of $\mathrm{QE}$. It is hardly surprising that all cells except one fall below 50\%; what is more remarkable is that there really is a fair proportion of cells with QE > 30\% and one cell with QE $>90 \%$ (this cell is analyzed further below). Figure 1(b) shows how the distri- 


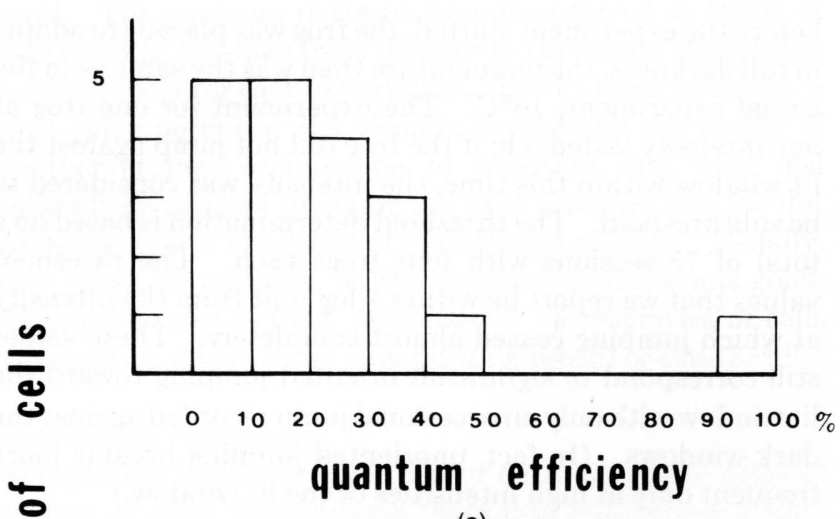

(a)

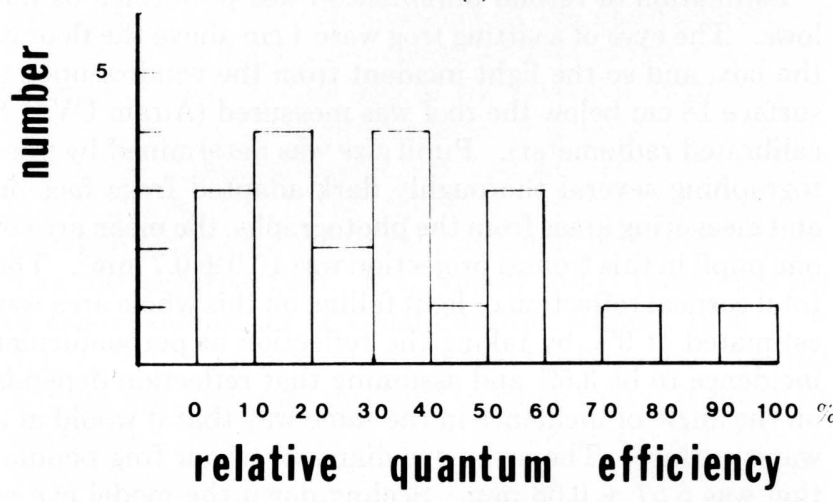

(b)

Fig. 1. Frequency histograms showing the numbers of ganglion cells (in a sample of 19 cells) falling into the indicated intervals of quantum efficiency for $50 \%$ detection of a flash of light. The values are based on a FOR estimation (see the Methods section), for each cell, of the threshold number of photoisomerizations $\left(N_{t}\right)$ and the number of equivalent-noise events $(X)$ within its summation area and time. (a) Estimated quantum efficiencies in relation to a perfect detector according to Eq. (5), i.e., $\mathrm{QE}=100 \times N_{t} /\left(N_{t}+X\right)$. (b) Estimated relative quantum efficiencies according to Eq. (6), i.e., $\mathrm{QE}_{\mathrm{rel}}=100 \times\left(N_{t}+D\right) /\left(N_{t}+X\right)$. This relates the threshold performance of the ganglion cell in darkness to that of a perfect detector, with the same summation parameters as the cell, that has to detect the flash isomerizations against a background rate of isomerizations equal to the rate of dark events in rods. (In this presentation the best cell technically gets an efficiency of $>100 \%$.)

bution changes if the dark events in $\operatorname{rods}^{6}$ is included as a real background of light affecting the threshold performance of the perfect detector [Eq. (3)]. The QE's of the cells relative to that limit appear considerably better, with no less than five cells above $50 \%$. Still, only 3 of these 19 cells had an estimated intrinsic noise low enough to be explicable by the rate of dark events in rods. ${ }^{12}$

The small proportion (15-20\%) of such sensitive, lownoise ganglion cells is in principle sufficient to ensure correspondingly sensitive behavior. ${ }^{12}$ A crucial question, however, considering the small number of such cells actually encountered, must be to what extent these FOR-based noise estimates actually reflect biological reality: whether the few low estimates might not just be the trivial lower tail of experimental variability.

The a priori reason to believe that the low estimates are essentially real is that FOR experiments in principle reflect any extra variability as flatter curves and hence higher, not lower, noise values. It is unlikely that, in an experiment involving a pseudorandom sequence of stimuli over $1-2 \mathrm{~h}$, a cell's responsiveness would fluctuate in phase with the stimulus sequence to produce a FOR curve that is much too steep. Yet it is obvious that even here experimental variation must occasionally give rise to underestimates.

\section{Three Other Response Properties Reflect Similar Noise Levels in Ganglion Cells}

We shall argue that the rate of equivalent dark events that we estimate by FOR experiments is biologically relevant because other important characteristics of the ganglion cells are consistent with an underlying noise of the same order of magnitude. Conversely, the agreement justifies the assumptions of how these other characteristics are determined.

We use three other easily accessible extracellular measures of the noise affecting the cell. First, there is the variation in the number of spikes in responses to dim suprathreshold flashes. Second, if it is postulated that the ganglion cell generates a spike if and only if the rate of rod events exceeds a criterion level, then the noise can be inferred from the rate of maintained discharge. Third, the dark light can

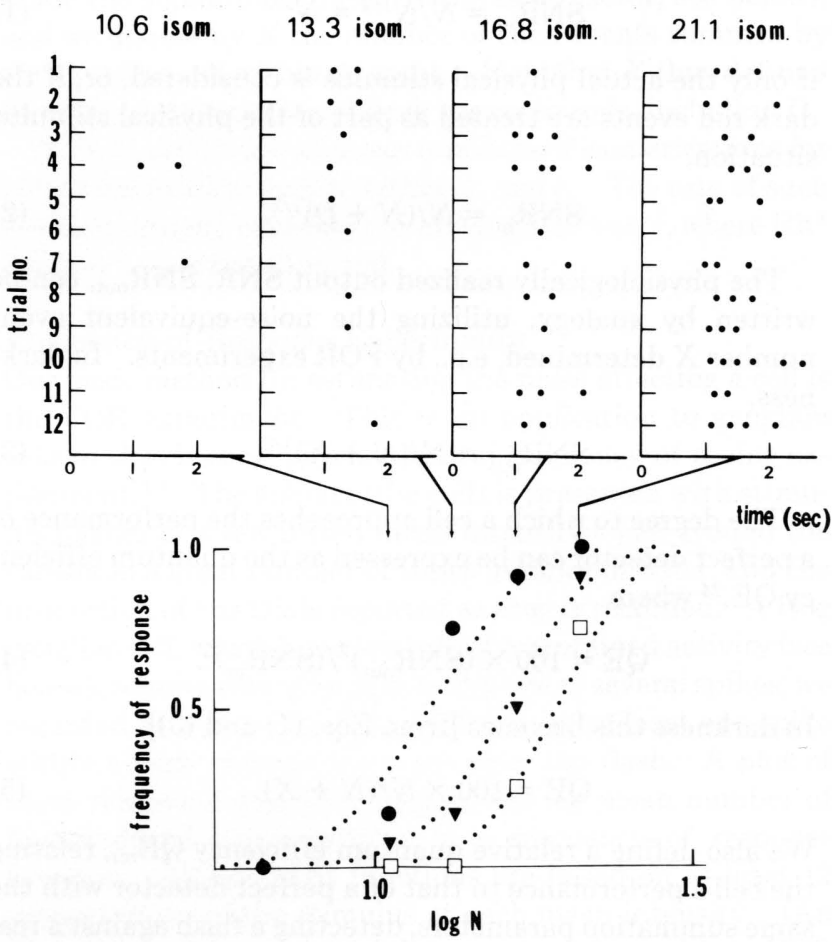

Fig. 2. Top, Manner in which the response discharges of one ganglion cell vary among 12 presentations of four fixed stimulus flashes. Each dot represents one spike potential; each row of dots is one discharge displayed on a time abscissa with the presentation of the 67 -msec flash at time zero. The mean numbers of photoisomerizations delivered to the receptive field of the cell by each flash are indicated above each set of records. Bottom, FOR functions extracted from these data: $\bullet$, the relative frequencies of responses containing one or more spikes; $\boldsymbol{\Delta}$, the relative frequencies of responses with two or more spikes; $\square$, the relative frequencies of responses with three or more spikes. The dotted curves are the FOR curves giving optimal fits to the above-mentioned three sets of data points; they correspond to the following threshold values and equivalent-noise values $\left(N_{t}, X\right):(11.6,0.3),(15.4,1.3)$, and $(19.2$, 0.1 ), respectively. The QSR of this cell was 4 (cell 1 in Table 1 ; cf. also Figs $3 \mathrm{~A}$ and $3 \mathrm{D})$. isom., Isomerizations. 


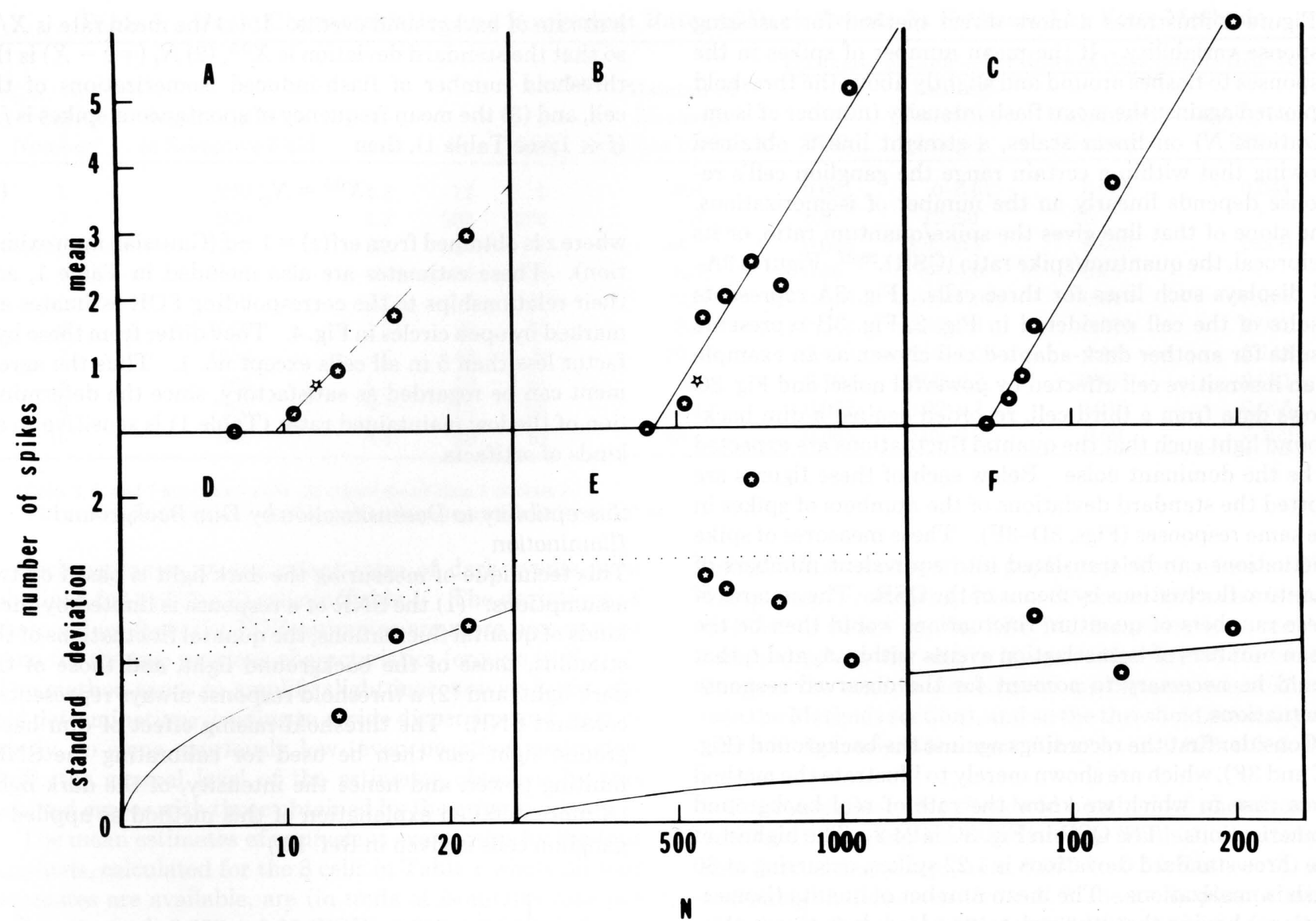

Fig. 3. The means and the standard deviations of the number of spikes in the responses of three cells, plotted as functions of $N$, the mean number of isomerizations induced by the stimulus flash, on linear coordinates. Each point (star within circle) is calculated from 12 presentations of the same stimulus. Data in A, D, B, and E are from dark-adapted cells (cells 1 and 2 in Table 1), and those in C and F are from a cell adapted by a steady background light from which the cell is estimated to sum 464 isomerizations within its summation area and one integration time. A, B, and C show the determination of the QSR. A straight line has been fitted to each set of points by linear regression; the reciprocal of the slope is the QSR. In addition, the figures show the $50 \%$ threshold numbers of isomerizations $N_{t}$ (stars in A and B); these are plotted at mean spike number 0.7 , since the Poisson probability $P(0)$ is $0.5(50 \%)$ for a mean of 0.7 . (In C, the threshold is not separately marked, because it coincides with the third data point from the left.) D, E, and F are plots of the standard deviations of the numbers of spikes in responses to intensities higher than $N_{t}$. (At lower intensities, when the mean number of spikes approaches 0 , the standard deviations, of course, also approach 0 and become meaningless for our purpose.) By the QSR the standard deviations can be translated into units of (equivalent) isomerizations, and vice versa (see the text). Thus the solid lines in D, E, and F show the values predicted from quantal fluctuations alone, the dashed line in $\mathrm{E}$ shows the prediction from the FOR estimate of the equivalent rate of dark events (in D that prediction is indistinguishable from the full-drawn line), and the dotted lines in $\mathrm{D}$ and $\mathrm{E}$ show the predictions from the two other estimates of the equivalent rate of dark events (the maintained activity and the susceptibility to background desensitization; cf. Table 1). (Note that a frog ganglion cell exposed to a background light puts down the gain so as to keep the maintained discharge at approximately the same low level as that observed in darkness: in C the maintained discharge was only 0.3 spike $/ 1000 \mathrm{sec}$, and so the probability of getting a spike within the usual 0.5-3-sec response window after the flash was negligible. As $t_{i}=1.7 \mathrm{sec}$ and $N_{t}=72$, the maintained discharge [according to Eq. (7)] suggests an underlying mean event number $X=385$, which is not so far from the 464 photoisomerizations expected from the background light within $A_{s}$ and $t_{i}$; see the text.)

be calculated from the cell's susceptibility to desensitization by dim backgrounds, according to a time-honored psychophysical rationale.

\section{Variability of Suprathreshold Discharges}

One way of assessing discharge variability is to generate FOR curves for different response criteria: one curve showing the proportion of trials giving one or more spikes, another for the proportion of trials giving two or more spikes, etc. (For a psychophysical analogy, see Ref. 4.) If the different response amplitudes are affected by the same noise source, the FOR functions optimized for the different response criteria should give the same noise estimate $X$ but different thresholds $N_{t}$.

As it is particularly important to know what to think about a cell with an estimated $\mathrm{QE}$ of $>90 \%$, we shall take that cell as an example. The top of Fig. 2 shows the whole set of responses to 12 presentations of four mean flash intensities; the bottom of Fig. 2 shows the FOR functions derived for response criteria of one, two, and three spikes. The dotted lines are maximum-likelihood Poisson curves, which correspond to parameter values $\left(N_{t}, X\right)=(11.6,0.3),(15.4,1.3)$, and $(19.2,0.1)$, respectively. The number of dark rod events ${ }^{6} D$ that this cell would be expected to sum within $A_{s}$ and $t_{i}$ is 1.6. The differences among the three $X$ estimates or between each of them and $D$ are not significant because the likelihoods of the FOR fits change little over this range. For example, the optimal fit $(X=0.3)$ to the points for the one-spike criterion has only 2.3 times higher likelihood than a curve with $X=D=1.6$. 
Figure 3 illustrates a more direct method for assessing response variability. If the mean number of spikes in the responses to flashes around and slightly above the threshold is plotted against the mean flash intensity (number of isomerizations $N$ ) on linear scales, a straight line is obtained showing that within a certain range the ganglion cell's response depends linearly on the number of isomerizations. The slope of that line gives the spike/quantum ratio, or its reciprocal, the quantum/spike ratio (QSR). ${ }^{26,27}$ Figures $3 \mathrm{~A}-$ $3 \mathrm{C}$ displays such lines for three cells: Fig. 3A represents results of the cell considered in Fig. 2, Fig. 3B represents results for another dark-adapted cell chosen as an example of an insensitive cell affected by powerful noise, and Fig. 3C shows data from a third cell, recorded against a dim background light such that the quantal fluctuations are expected to be the dominant noise. Below each of these figures are plotted the standard deviations of the numbers of spikes in the same responses (Figs. 3D-3F). These measures of spike fluctuations can be translated into equivalent numbers of quantum fluctuations by means of the QSR. The squares of these numbers of quantum fluctuations would then be the mean numbers of isomerization events within $A_{s}$ and $t_{i}$ that would be necessary to account for the observed response fluctuations.

Consider first the recordings against the background (Fig. $3 \mathrm{C}$ and $3 \mathrm{~F}$ ), which are shown merely to illustrate the method for a case in which we know the rate of real background isomerizations. The QSR in Fig. 3C is 24.2. The highest of the three standard deviations is 1.22 spikes, occurring at 80 flash isomerizations. The mean number of quanta (isomerizations) having the appropriate standard deviation is then $(24.2 \times 1.22)^{2}=872$. Of these, 80 belong to the stimulus, leaving 792 background isomerizations. This is an upper estimate. A lower estimate, 344 , is obtained by performing the corresponding calculation for the smallest response standard deviation, 0.90 at 130 flash isomerizations. The range $344-792$ is an adequate, although not very precise, estimate of the 464 photoisomerizations that this cell is, in fact, calculated to sum from the known background light within $A_{s}$ and $t_{i}$, suggesting that its response variability is indeed determined by quantal fluctuations. (The standard deviation expected from 464 events is plotted as a continuous line in Fig. 3F; for an explanation of the lines in Figs. 3D-3E, see the caption to Fig. 3.)

We then use the same rationale to estimate the dark background that would account for the response variability in the dark-adapted cells. In Table 1 the results from all 10 cells thus investigated are collected for comparison with the estimates of equivalent-event rate based on optimized FOR fits. It can be seen that in all cells there is a basic agreement as regards orders of magnitude, and in all but three cases the FOR estimate actually falls inside the range of values that account for discharge variability. In Fig. 4, the filled circles plot the relation between the two estimates for the cells in Table 1.

\section{The Maintained Discharge}

If the assumption underlying the FOR experiments, that the ganglion cell is a counter of isomerization events falling within $A_{s}$ and $t_{i}$ and produces a spike if and only if the number exceeds a criterion $c$, is applied to the maintained discharge, this allows yet another estimation of an equiva- lent rate of background events. If (1) the mean rate is $X / t_{i}$, so that the standard deviation is $X^{0.5},(2) N_{t}(=c-X)$ is the threshold number of flash-induced isomerizations of the cell, and (3) the mean frequency of spontaneous spikes is $f / t_{i}$ ( $f \ll 1$; see Table 1$)$, then

$$
z X^{0.5}=N_{t},
$$

where $z$ is obtained from $\operatorname{erf}(z)=1-f$ (Gaussian approximation). These estimates are also included in Table 1, and their relationships to the corresponding FOR estimates are marked by open circles in Fig. 4. They differ from these by a factor less than 5 in all cells except no. 1 . Thus the agreement can be regarded as satisfactory, since the determination of the low maintained rates (Table 1) is sensitive to all kinds of artifacts.

\section{Susceptibility to Desensitization by Dim Background Illumination}

This technique of measuring the dark light is based on two assumptions: (1) the SNR of a response is limited by three kinds of quantal fluctuations, the quantal fluctuations of the stimulus, those of the background light, and those of the dark light; and (2) a threshold response always represents a constant SNR. The threshold-raising effect of dim background light can then be used for calibrating the SNRlimiting power, and hence the intensity, of the dark light. (A more detailed explanation of this method as applied to ganglion cells is given in Ref. 10.)

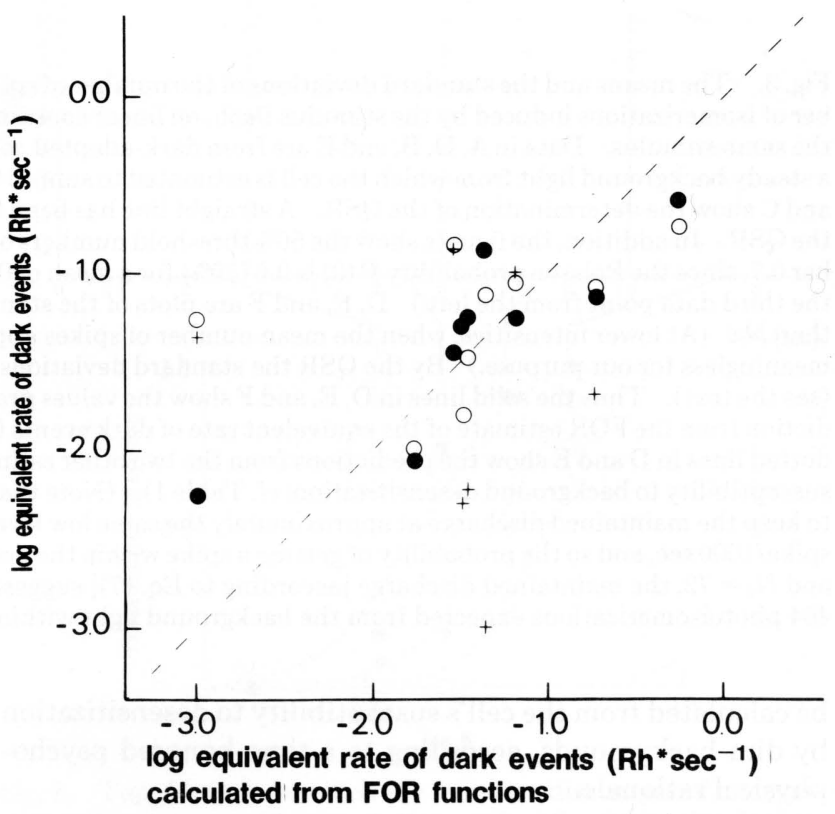

Fig. 4. A comparison of the equivalent rate of dark events (in units of $\mathrm{Rh}^{*} \mathrm{sec}^{-1}$ ) as estimated by, on one hand, the optimization of frequency of response functions (abscissa) and, on the other hand, three other methods (ordinate): $\bullet$, the variability of discharges; $\bigcirc$, the maintained activity; + , the susceptibility to densensitization by backgrounds. (The values derived from the variability of discharges have been calculated as midpoints between the logarithms of the upper and lower estimates given in Table 1.) A $45^{\circ}$ dashed line indicating perfect correlation has been drawn for visual guidance. Only the background-based estimates show no correlation with the FOR estimates, but they still indicate noise of the same order of magnitude as the other methods. This figure is a graphic summary of the noise estimates in Table 1 (cell 2 was excluded, as its large noise was far outside the range of the others). 
Table 1. Basic Characteristics and Equivalent Rates of Dark Events As Estimated by Four Methods

\begin{tabular}{|c|c|c|c|c|c|c|c|c|c|}
\hline \multirow[b]{2}{*}{$\begin{array}{c}\text { Cell } \\
\text { Number }^{a}\end{array}$} & \multirow[b]{2}{*}{$\begin{array}{l}\text { Number of Rods } \\
\text { in Receptive Field }\end{array}$} & \multirow[b]{2}{*}{$\begin{array}{c}t_{i} \\
(\mathrm{sec})\end{array}$} & \multirow[b]{2}{*}{$N_{t}$} & \multirow[b]{2}{*}{ QSR } & \multirow[b]{2}{*}{$\begin{array}{c}\text { Maintained } \\
\text { Discharge } \\
\text { (spikes/1000 sec) }\end{array}$} & \multicolumn{4}{|c|}{ Estimates of Equivalent Rates of Dark Events $\left(\mathrm{Rh}^{*} \mathrm{sec}^{-1}\right)$} \\
\hline & & & & & & FOR & $\begin{array}{c}\text { Discharge } \\
\text { Variability } \\
\text { (range) }\end{array}$ & $\begin{array}{c}\text { Maintained } \\
\text { Discharge }\end{array}$ & $\begin{array}{c}\text { Background } \\
\text { Effect }\end{array}$ \\
\hline 1 & 230 & 1.2 & 12 & 4 & 0.8 & 0.001 & $0-0.01$ & 0.055 & 0.044 \\
\hline 2 & 940 & 1.2 & 565 & 124 & 1.8 & 8.8 & $12-60$ & 35 & $?$ \\
\hline 3 & 1510 & 2.1 & 24 & 7 & 44 & 0.035 & $0.03-0.1$ & 0.033 & 0.006 \\
\hline 4 & 1510 & 1.3 & 52 & 36 & 2.5 & 0.56 & $0.1-0.6$ & 0.19 & $?$ \\
\hline 5 & 250 & 2.7 & 7 & 1.5 & 2.3 & 0.018 & $0.006-0.012$ & 0.01 & -0.001 \\
\hline 6 & 1060 & 1.7 & 29 & 15 & 3.8 & 0.044 & $0.05-0.4$ & 0.075 & 0.001 \\
\hline 7 & 1850 & 1.7 & 45 & 9 & 10 & 0.029 & $0.02-0.06$ & 0.15 & 0.142 \\
\hline 8 & 160 & 1.5 & 13 & 11 & 1.3 & 0.19 & $0.02-0.3$ & 0.083 & 0.021 \\
\hline 9 & 750 & 2.7 & 15 & 16 & 1.4 & 0.032 & $0.02-0.1$ & 0.016 & 0.005 \\
\hline 10 & 950 & 2.1 & 20 & 10 & 33 & 0.065 & $0.02-0.2$ & 0.09 & 0.102 \\
\hline
\end{tabular}

${ }^{a}$ Cells 3,4 , and 7 are class 3 cells; all others are of class 1 or class 2.

${ }^{b}$ The expected rate of dark events in rods at this temperature is $0.006 \mathrm{Rh}^{*} \mathrm{sec}^{-1}$.

Such estimates of equivalent rates of dark events were obtained for 8 of the 10 cells in Table 1 . The deviations of these values from the FOR estimates are more pronounced than for the two previous characteristics (crosses in Fig. 4). The method tends to amplify slight inaccuracies in threshold determinations, leading to a wide dispersion and, particularly, to some spuriously low (even negative) estimates. Still, the general level of the estimates obtained by this method agrees with those obtained by the other methods.

The mean estimates of equivalent event rates by the four methods, calculated for the 8 cells in Table 1 where all four estimates are available, are (in units of isomerizations per rod per second) $0.052 \pm 0.02$ (FOR), $0.052 \pm 0.01$ (discharge variability), $0.064 \pm 0.02$ (maintained discharge), and 0.040 \pm 0.02 (background effect).

In summary, it does seem that the equivalent rate of dark events estimated by optimization of FOR curves is a biologically meaningful entity, consistent with other functional characteristics of the cells. A corollary is that one can feel some confidence also in the low estimates, suggesting that the reliability of a small proportion of sensitive cells is limited by an intrinsic noise level not significantly higher than that represented by the dark rod events described by Baylor et al. ${ }^{6}$ We now turn to the question of how the absolute behavioral sensitivity of the intact animal is related to that of the retinal ganglion cells.

\section{The Absolute Sensitivity of Visually Guided Behavior: Phototaxis}

Jumping toward a lit window is about as close to expressing simple psychophysical light detection as a frog can get. Besides the perception of light in a given direction, no further information is required for the task. Still, it should be realized that many factors affect the frog's willingness to jump, and so the absence of jumping does not prove that the frog has not seen the light. The behavioral thresholds can at best give lower limits to visual sensitivity, and so it is not surprising that the same type of experiments yielded somewhat different thresholds in Moscow and in Helsinki. These threshold values, which still corresponded to highly significant jumping responses, were the equivalents of 41 and $67 \mathrm{q}_{502} \mathrm{~mm}^{-2} \mathrm{sec}^{-1}$ at the cornea, respectively. For a dark-adapted pupil size of $10 \mathrm{~mm}^{2}$ (as projected onto the frontal plane; see the Methods section) and a corneal reflec- tion of $9 \%$ (across the whole pupil projection), a total of 370$610 \mathrm{q}_{502} \mathrm{sec}^{-1}$ reach the retina. Of these, 36\% cause isomerizations in rods, ${ }^{21}$ giving $133-220$ isomerizations $\sec ^{-1}$ as summed across the entire image. The size of the retinal image of the window viewed at $18-\mathrm{cm}$ distance was $0.92 \mathrm{~mm}^{2}$ (see the Methods section), and so the threshold intensity was 145-240 isomerizations $\mathrm{mm}^{-2} \mathrm{sec}^{-1}$, or $0.009-0.015 \mathrm{Rh}^{*}$ $\mathrm{sec}^{-1}$.

For comparison, the threshold numbers of photoisomerizations of the ganglion cells listed in Table 1 , recalculated into intensities, range from 0.007 to $0.50 \mathrm{Rh}^{*} \mathrm{sec}^{-1}$, and, excluding the less-sensitive cell 2 , the mean plus or minus the standard error is $0.021 \pm 0.006 \mathrm{Rh}^{*} \mathrm{sec}^{-1}$.

\section{DISCUSSION}

\section{The Intrinsic Retinal Noise Affecting Ganglion-Cell Performance}

We find satisfactory agreement among four different estimates of the noise affecting dark-adapted ganglion cells in the frog retina. All four estimation techniques, however, must employ the estimated sensitivity of the cell: the noise estimations from FOR functions, from the sensitivity-depressing power of backgrounds, and from the maintained discharge explicitly use the threshold number of isomerizations $N_{t}$, while the estimation from the discharge variability depends on the sensitivity level by means of the QSR. The problem is that, unless the actual mean numbers of signal photoisomerizations involved are known with confidence, a high response variance relative to the mean can be interpreted in two alternative ways. Either there are few isomerizations altogether, whereby the relative Poisson variance of the quantal fluctuations is high, or there are many isomerizations but also intrinsic noise. Could it be that high estimates of intrinsic noise are obtained (when they are obtained) only because the number of photoisomerizations involved is overestimated?

It is worth noting that this argument cannot cast doubt on the existence of the most sensitive and reliable cells, where the known dark events in rods can in principle account for most of the intrinsic noise. Our calibrations of light intensities, absorption in the rod mosaic, etc. have been meticulously checked, ${ }^{10}$ and so no cell has received significantly more isomerizations than we thought. The existence of such cells 
is the more remarkable because the noise expected from spontaneous dark events in frog rods at $11.5^{\circ} \mathrm{C}$ is so low as to be of the same order of magnitude as that physical lower limit, the Johnson noise, which is necessarily connected with the resistive properties of rods. [See, e.g., Ref. 28, p. 288. A simple calculation for a single rod yields a Johnson current (rms) of a few femtoamperes in the relevant frequency band. If spontaneous dark events with amplitudes ${ }^{29}$ of $0.5-1 \mathrm{pA}$ occur at a rate of $0.006 \mathrm{sec}^{-1}$, and the integration time is 2 sec, then the current that they produce is $\sim 10 \mathrm{fA}$. We are indebted to Govardovskii ${ }^{30}$ for pointing out this to us.]

However, we are faced with the opposite problem: do some ganglion cells, in fact, have access to fewer quanta than we thought? The possibility that the effective number of isomerizations could be small has been put forward by Cohn et al., ${ }^{31}$ who carried out a receiver operating characteristic analysis of dimming detection ganglion cells in the retina of Rana pipiens. Cohn et al. were forced to conclude that ganglion cells are connected with only $0.1-1.6 \%$ of the rods within their receptive fields, in order to reconcile apparent perfect quantum detector performance with high relative noise. These authors did not perform their experiments at the absolute threshold but instead at levels of steady background illumination where photon fluctuations dominate over dark rod events. Their main conclusion, that ganglion cells under such conditions can approach perfect detector performance, is in agreement with our results ${ }^{9}$ (see also Figs. $3 \mathrm{C}$ and $3 \mathrm{~F}$ of the present study). However, we find the hypothesis of extremely sparse rod connectivity unlikely. First, extensive electrical coupling between rods in the toad retina has been well documented ${ }^{32-35}$; second, rod bipolar cells, at least in goldfish, are known to be coupled directly to all the rods within their dendritic domain. ${ }^{36}$ Further, we have found that in Rana temporaria the off responses of the dimming detectors are quite as sensitive as the on responses of sensitive cells in this study. A rod connectivity of 0.1$1.6 \%$ would lead to the absurd conclusion that such cells could respond reliably to the turning off of lights, producing, on an average, only $0.1-1$ isomerization within the summation time and area.

We think that the results of Cohn et al..$^{31}$ indicate, instead, substantial intrinsic retinal noise connected with the transduction and transmission of photon signals. Such multiplicative noise has precisely the effect of retaining the Poisson structure of underlying events while misleadingly suggesting low quantum efficiency (i.e., that a low fraction of quanta is utilized by the cell). ${ }^{37}$

It is also noteworthy that, although there is a correlation between high equivalent noise and low sensitivity (as is to be expected), one does not suffice to explain the other. In fact, threshold responses of insensitive cells are, on the average, much more reliable (have a higher SNR) than those of sensitive cells. ${ }^{11,12}$ Clearly, if noise estimates were simple functions of estimated sensitivity, this would not be the case. (An illustration: erroneous calibration of the absolute intensity would imply an apparent shift of a FOR function to higher $\log N$ values but could not explain the observation that less sensitive cells show steeper FOR functions. ${ }^{11,12}$ )

We conclude that besides a small fraction of extremely reliable and sensitive cells, there really are many cells that are affected by retinal noise more powerful by orders of magnitude. Where does that noise come from? There are at least two conceivable sources of extra noise: (1) the large- ly antagonistic or inhibitory connections subserving more specific processing by the ganglion cells ${ }^{9} ;(2)$ the variation in the amplitude of isomerization signals as seen by the ganglion cell (multiplicative noise ${ }^{37}$ ), which is due both to variation in the amplitude of the initial receptor event ${ }^{29}$ and to the varying strength of the synaptic connection of the ganglion cell to different parts of its (Gaussian ${ }^{18}$ ) receptive field.

\section{The Relation between the Behavioral Threshold and the Performance of Retinal Ganglion Cells}

The behavioral threshold (in Moscow) corresponded to $0.009 \mathrm{Rh}^{*} \mathrm{sec}^{-1}$ over the retinal image of the window $(\sim 0.92$ $\mathrm{mm}^{2}$ ). A simple way of relating this to the performance of the ganglion cells is to consider how the cells in Table 1 would respond to such illumination. It can be seen that in 2 of the 10 cells (cells 3 and 9) the 50\% response threshold would be exceeded. It is noteworthy that these are not the cells with the lowest estimated noise but cells with rather large receptive fields and longer integration times. Thus the absolute sensitivity of this behavior cannot be considered to be strictly limited by the dark events in rods.

The distribution of thresholds $\left(N_{t}\right)$ and summation areas among 105 dark-adapted ganglion cells (with summation areas $>0.01 \mathrm{~mm}^{2}$ ) is given by Donner ${ }^{12}$ (Fig. 6 in that paper). If a common integration time of $2.1 \mathrm{sec}$ (the mean value for dark-adapted frog ganglion cells at $\left.11^{\circ} \mathrm{C}\right)^{15}$ is applied, it is found that $0.009 \mathrm{Rh}^{*} \mathrm{sec}^{-1}$ exceeds the $50 \%$ response threshold in 22 of the 105 cells (i.e., these cells would give one or more spikes per second of integration time). From the fact that $22 / 105$, i.e., some $20 \%$ of all cells with $A_{s}>0.01 \mathrm{~mm}^{2}$, respond to $0.009 \mathrm{Rh}^{*} \mathrm{sec}^{-1}$, one can conclude that the responding cells represent at least a threefold coverage of the visual field. ${ }^{12}$ Thus the phototactic jumping behavior apparently requires a fairly reliable signal for initiation, i.e., rather massive activity in a subpopulation of ganglion cells.

In a more general sense, the behavioral sensitivity level found here may still be dependent on the low rate of dark rod events. Our measured absolute sensitivity in the frog is higher than most other estimates from any species. Preliminary experiments by us on the absolute sensitivity of humans in a situation comparable with that used for the frogs (subject freely scanning with both eyes a region where an extended stimulus of long duration appears) suggest a threshold of about 300 isomerizations $\mathrm{sec}^{-1} \mathrm{~mm}^{-2}$ of retina for humans, to be compared with the lower estimate of 145 isomerizations $\mathrm{sec}^{-1} \mathrm{~mm}^{-2}$. The classical work by Denton and Pirenne ${ }^{38}$ on the melanophore expansion reflex in Xenopus laevis gave an absolute threshold equivalent to $\sim 2900$ $\mathrm{q}_{502} \mathrm{~mm}^{-2} \mathrm{sec}^{-1}$ incident upon the retina, about 7 times higher than our threshold of $\sim 400 \mathrm{q}_{502} \mathrm{~mm}^{-2} \mathrm{sec}^{-1}$. Experiments by Powers and Easter ${ }^{39}$ with goldfish, based on conditioning of breathing and heart rates, yielded absolute thresholds of 400-830 isomerizations $\mathrm{sec}^{-1}$, while our lower threshold estimate for frogs corresponds to 133 isomerizations $\sec ^{-1}$. Powers and Easter also recalculate into the same units the value obtained by Denton and Pirenne ${ }^{40}$ for the human absolute threshold to an extended stimulus of long duration, arriving at 900 isomerizations $\mathrm{sec}^{-1}$.

Thus the absolute visual sensitivity that we have found in frogs jumping toward a lit window is higher than all these comparable estimates in other vertebrates. In lower vertebrates, it is hard to assess to what extent differences are due to different techniques. However, the greater sensitivity of 
frogs as compared with humans is interesting. For a frog at $16^{\circ} \mathrm{C}$, the expected rate of dark rod events is $160 \mathrm{~mm}^{-2} \mathrm{sec}^{-1}$; for a human at $37^{\circ} \mathrm{C}$ the expected rate is on the order of 1000 $\mathrm{mm}^{-2} \mathrm{sec}^{-1}$. Raising the frog's temperature from $16^{\circ} \mathrm{C}$ to $37^{\circ} \mathrm{C}$ would (by extrapolation of Fig. 9 in Ref. 6) increase the dark event rate 16 -fold. It is instructive to see how such noise would affect the 22 cells in the sample of 105 cells referred to above that would respond to the behavioral threshold intensity. At $11^{\circ} \mathrm{C}$ their $\mathrm{SNR}_{\text {in }}$ values at the $50 \%$ threshold $\left[\mathrm{SNR}_{\text {in }}\right.$ according to Eq. (2), i.e., taking the dark rod events $D$ as real isomerizations] were distributed as follows: three cells above $\mathrm{SNR}_{\text {in }}=4$, four cells with values between 3 and 4, six cells with values between 2 and 3, and nine cells with values between 1 and 2 . Under the assumption that the cells do not reset their $50 \%$ response threshold $N_{t}$ or increase their spatiotemporal summation, putting the $D$ value appropriate for $37^{\circ} \mathrm{C}$ into Eq. (2) yields $\mathrm{SNR}_{\text {in }}$ values below 1 for all but one of the 22 cells! The dark events in rods probably form the noise component with the strongest temperature dependence. Even if not strictly limiting at low temperatures, they may quickly become so as the temperature is raised.

\section{ACKNOWLEDGMENTS}

This work was supported by grants (joint project no. 22) from the Academy of Sciences of the USSR and the Academy of Finland to O. Yu. Orlov and T. Reuter, from the Academy of Finland to T. Reuter, from Ella och Georg Ehrnrooths stiftelse to K. Donner, and from Societas Scientarum Fennica to T. Reuter and K. Donner. We wish to thank V. I. Govardovskii for many helpful discussions.

\section{REFERENCES}

1. G. T. Fechner, Elemente der Psychophysik (Breitkopf \& Härtel, Leipzig, 1860).

2. R. B. Barnes and M. Czerny, "Lässt sich ein Schroteffekt der Photonen mit dem Auge beobachten?" Z. Physik 79, 436-449 (1932).

3. S. Hecht, S. Shlaer, and M. H. Pirenne, "Energy, quanta, and vision,” J. Gen. Physiol. 25, 819-840 (1942).

4. H. B. Barlow, "Retinal noise and absolute threshold," J. Opt. Soc. Am. 46, 634-639 (1956).

5. J. F. Ashmore and G. Falk, "Dark noise in retinal bipolar cells and stability of rhodospin rods," Nature 270, 69-71 (1977).

6. D. A. Baylor, G. Matthews, and K.-W. Yau, "Two components of electrical dark noise in toad retinal rod outer segments," J. Physiol. 309, 591-621 (1980).

7. D. A. Baylor, B. J. Nunn, and J. F. Schnapf, "The photocurrent, noise and spectral sensitivity of rods of the monkey Macaca fascicularis," J. Physiol. 357, 575-607 (1984).

8. H. B. Barlow, "Increment thresholds at low intensities considered as signal/noise discriminations," J. Physiol. 136, 469-488 (1957).

9. T. Reuter, K. Donner, and D. R. Copenhagen, "Does the random distribution of discrete photoreceptor events limit the sensitivity of the retina?" Neurosci. Res. Suppl. 4, 163-180 (1986).

10. D. R. Copenhagen, K. Donner, and T. Reuter, "Ganglion cell performance at absolute threshold in toad retina: effects of dark events in rods," J. Physiol. (to be published).

11. K. Donner, C. Hydén, and T. Reuter, "Noise and absolute threshold of frog retinal ganglion cells," Acta Univ. Oul. A 179, 35-38 (1986).

12. K. Donner, "The absolute sensitivity of vision: can a frog become a perfect detector of light-induced and dark rod events?" Phys Scr. (to be published).

13. K. O. Donner and T. Reuter, "Visual adaptation of the rhodospin rods in the frog's retina,” J. Physiol. 199, 59-87 (1968).
14. A.-C. Bäckström, S. Hemilä, and T. Reuter, "Directional selectivity and colour coding in the frog retina," Med. Biol. 56, 72-83 (1978).

15. K. Donner, "Adaptation-related changes in the spatial and temporal summation of frog retinal ganglion cells," Acta Physiol. Scand. (to be published).

16. H. R. Maturana, J. Y. Lettvin, W. H. Pitts, and W. McCulloch, "Physiology and anatomy of vision in the frog," J. Gen. Physiol. 43, 385-404 (1960).

17. A.-C. Bäckström and T. Reuter, "Receptive field organization of ganglion cells in the frog retina: contributions from cones, green rods and red rods," J. Physiol. 246, 79-107 (1975).

18. K. Donner and M.-L. Grönholm, "Center and surround excitation in the receptive fields of frog retinal ganglion cells," Vision Res. 24, 1807-1819 (1984).

19. B. G. Cleland and C. Enroth-Cugell, "Quantitative aspects of sensitivity and summation in the cat retina," J. Physiol. 198, 1738 (1968).

20. K. Donner, "Receptive fields of frog retinal ganglion cells: response formation and light-dark-adaptation," J. Physiol. 319, 131-142 (1981).

21. S. Hemilä and T. Reuter, "Longitudinal spread of adaptation in the rods of the frog's retina," J. Physiol. 310, 501-528 (1981).

22. D. A. Baylor and A. L. Hodgkin, "Detection and resolution of visual stimuli by turtle photoreceptors," J. Physiol. 234, 163198 (1973).

23. H. B. Barlow, "Retinal and central factors in human vision limited by noise," in Vertebrate Photoreception, H. B. Barlow and P. Fatt, eds. (Academic, London, 1977), pp. 337-358.

24. A. Rose, "Vision: human versus electronic," in Vertebrate Photoreception, H. B. Barlow and P. Fatt, eds. (Academic, London, 1977), pp. 1-13.

25. J. S. du Pont and P. J. de Groot, "A schematic dioptic apparatus for the frog's eye (Rana esculenta)," Vision Res. 16, 803-810 (1974).

26. H. B. Barlow and W. R. Levick, "Three factors limiting the reliable detection of light by retinal ganglion cells of the cat," J. Physiol. 200, 1-24 (1969).

27. H. B. Barlow, W. R. Levick, and M. Yoon, "Responses to single quanta of light in retinal ganglion cells of the cat," Vision Res. Suppl. 3, 87-101 (1971).

28. P. Horowitz and W. Hill, The Art of Electronics (Cambridge U. Press, Cambridge, 1980).

29. D. A. Baylor, T. D. Lamb, and K.-W. Yau, "Responses of retinal rods to single photens," J. Physiol. 288, 613-634 (1979).

30. V. I. Govardovskii, "Intrinsic noise, threshold sensitivity, and adaptation in the retina," in Sensory Systems (Nauka, Leningrad, 1978), pp. 61-81 (in Russian).

31. T. E. Cohn, D. R. Green, and W. P. Tanner, Jr., "Receiver operating characteristic analysis. Application to the study of quantum fluctuation effects in optic nerve of Rana pipiens," J. Gen. Physiol. 66, 583-616 (1975).

32. G. L. Fain, "Quantum sensitivity of rods in the toad retina," Science 187, 838-841 (1975).

33. H. F. Leeper, R. A. Normann, and D. R. Copenhagen, "Evidence for passive electrotonic interactions in red rods of toad retina," Nature 275, 234-236 (1978).

34. G. H. Gold, "Photoreceptor coupling in retina of the toad, Bufo marinus. II. Physiology," J. Neurophysiol. 42, 311-328 (1979).

35. E. R. Griff and L. H. Pinto, "Interactions among rods in the isolated retina of Bufo marinus," J. Physiol. 314, 327-354 (1981).

36. A. T. Ishida, W. J. Stell, and D. O. Lightfoot, "Rod and cone inputs to bipolar cells in goldfish retina," J. Comp. Neurol. 191, 315-335 (1980).

37. P. G. Lillywhite, "Multiplicative intrinsic noise and the limits to visual performance,” Vision Res. 21, 291-296 (1981).

38. E. J. Denton and M. H. Pirenne, "The visual sensitivity of the toad Xenopus laevis,” J. Physiol. 125, 181-207 (1954).

39. M. K. Powers and S. S. Easter, Jr., "Absolute visual sensitivity of the goldfish," Vision Res. 18, 1137-1147 (1978).

40. E. J. Denton and M. H. Pirenne, "The absolute sensitivity and functional stability of the human eye," J. Physiol. 123, 417-442 (1954). 